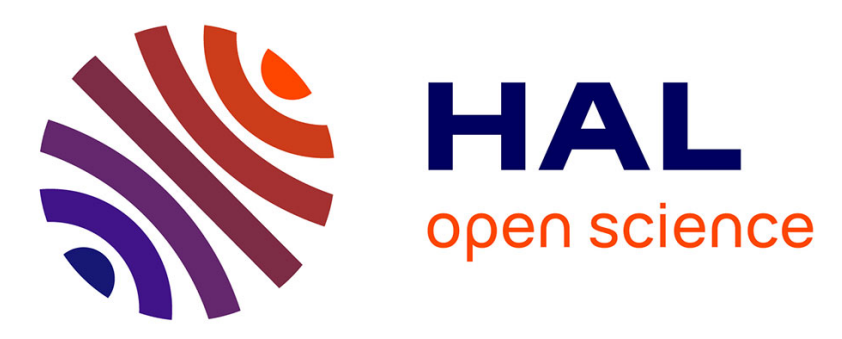

\title{
Evidential calibration process of multi-agent based system: An application to forensic entomology
}

Alexandre Veremme, Éric Lefevre, Gildas Morvan, Daniel Dupont, Daniel Jolly

\section{To cite this version:}

Alexandre Veremme, Éric Lefevre, Gildas Morvan, Daniel Dupont, Daniel Jolly. Evidential calibration process of multi-agent based system: An application to forensic entomology. Expert Systems with Applications, 2012, 39 (3), pp.2361-2374. 10.1016/j.eswa.2011.08.075 . hal-03331898

\section{HAL Id: hal-03331898 \\ https://hal.science/hal-03331898}

Submitted on 2 Sep 2021

HAL is a multi-disciplinary open access archive for the deposit and dissemination of scientific research documents, whether they are published or not. The documents may come from teaching and research institutions in France or abroad, or from public or private research centers.
L'archive ouverte pluridisciplinaire HAL, est destinée au dépôt et à la diffusion de documents scientifiques de niveau recherche, publiés ou non, émanant des établissements d'enseignement et de recherche français ou étrangers, des laboratoires publics ou privés. 


\title{
Evidential calibration process of multi-agent based system: an application to forensic entomology
}

\author{
Alexandre Veremme ${ }^{(1,2,3)}$, Éric Lefevre ${ }^{(1,2)}$, Gildas Morvan ${ }^{(3)}$, \\ Daniel Dupont ${ }^{(3)}$, Daniel Jolly ${ }^{(1,2)}$ \\ (1) Université Lille Nord de France, F-59000 Lille France \\ (2) U-Artois, LGI2A EA 3926 \\ Technoparc Futura, F-62400 Béthune, firstname.name@univ-artois.fr \\ (3) HEI, Département Ingénierie et Sciences du Vivant \\ 13 rue de Toul, F-59800 Lille, firstname.name@hei.fr
}

\begin{abstract}
Forensic entomology consists, during a criminal investigation, in studying the insects found on a cadaver to estimate the time of death. This is the only technique that can be used for a large post-morten interval. But, because of the important system complexity, the result given by the expert are imperfect. In this paper, a Decision Support System (DSS) has been developed to take into account all the ecosystemic parameters and a significant quantity of biological models. The proposed DSS is based on the belief function theory to validate and calibrate agent based simulations. First results of this architecture are presented within the framework of a real forensic examination.
\end{abstract}

Keywords: Multi-agent based simulation, Evidence theory, Dempster-Shafer theory of belief function, Forensic entomology, Validation, Calibration.

\section{Introduction}

Modeling biological and living systems, often considered as complex systems, with a large number of heterogeneous individuals interacting, is not an obvious exercise. Various paradigms can be used, but intuitively, the multi-agent based paradigm [1] seems to be an ideal alternative, particularly to enable property emergence and self-organization from individual interactions. While the computational cost of running a multi-agent based simulation can be exorbitant, it increases significantly when a reasoning or treatment is desired from these simulations.

In particular, in order to define a "good" model, i.e. able to correctly simulate a given real system, two generic steps, conceptually distinct, are conducted : validation and calibration [2]. Simulation validation consists in measuring or determining if the simulation is "reasonably" similar to a given reality. The idea is then to compare the data obtained from the simulation to their counterparts 
in reality. As for it, calibration is an iterative process, upstream from the validation, consisting in :

1. Determining the model parameters,

2. Executing and validating the simulation,

3. And, function of the obtained results, choosing other parameter values and coming back to step 1 .

4. Stopping when the simulation is enough similar to the real system.

It is often necessary to validate the simulation through observations ; in multi-agent based simulations, these observations are usually made from local agent properties, the global simulation properties are not accessible or available.

The problem is that these data are, by definition in complex systems, numerous, and their units and types (e.g. qualitative or quantitative) are different. In this context, the proximity determination of a simulation from a reality can be difficult, particularly because the quality of their information may be very imperfect (i.e. uncertain, imprecise). It seems interesting to use and develop a specific formalism to represent and manage such information. Among the existing theories, the theory of belief functions $[3,4]$ can be well adapted to this kind of problem $[5,6]$.

In the framework of developing a decision support system (DSS) dedicated to forensic medicine, we face the problem of calibration and validation of multiagent based simulations. The aims of this global DSS are to determine the time of death of a cadaver, i.e. calibrate the time parameter. This is done by validating simulations by comparing their proximity to a reality provided by experts - i.e. a set of biological data measured at a crime scene -. We develop a pyramidal validation system of multi-agent simulations based on the belief function theory and included in a general calibration system.

In the first part of this document ( $c f$. sub-section 2.1), the belief function theory is presented. In the next sub-section 2.2, foundations of multi-agent systems are briefly presented. The sequel of the article is dedicated to the validation (section 3) and calibration (section 4) of agent based simulations in the framework of the belief function theory. First results are then presented in the forensic application (section 5) before developing discussions and conclusions (section 6).

\section{Background}

The belief function theory, also called evidence theory, was introduced by Dempster [7] during his work on the lower and upper bounds of a distribution probability family. The initial theory was modified and ameliorated on several occasions, for instance through the work of Shafer [3] then later thanks to the work of Philippe Smets on the transferable belief model (the TBM), a nonprobabilistic interpretation of the evidence theory. We present in this part, the main concepts of the belief function theory. For more details, the interested reader may refer to [4]. 


\subsection{Belief function theory}

\subsubsection{Basic concepts}

Let $\Omega$ be the exclusive set of $K$ hypotheses, solution of a given problem. $\Omega$ is called the frame of discernment and is defined as follows:

$$
\Omega=\left\{\omega_{1}, \omega_{2}, \ldots, \omega_{K}\right\}
$$

From this frame of discernment $\Omega$, the power set $2^{\Omega}$ can be built, including the $2^{K}$ proposals $A$ of $\Omega$ :

$$
2^{\Omega}=\{A \mid A \subseteq \Omega\}
$$

A mass function (or allocation ${ }^{1}$ ), noted $m$, is defined by $2^{\Omega}$ in $[0,1]$, and verifies:

$$
\sum_{A \subseteq \Omega} m(A)=1
$$

Each subset $A \subseteq \Omega$ such that $m(A) \neq 0$ is called a focal element of $m$. Thus, mass $m(A)$ represents the degree of belief allocated to the proposal $A$ and that cannot, in the present state of knowledge, be attributed to a more specific subset than $A$. A BBA $m$ is said to be dogmatic if $\Omega$ is not a focal set and normal if $m(\emptyset)=0$. As an example, in the transferable belief model of Philippe Smets, the condition $\sum_{\emptyset \neq A \subseteq \Omega} m(A)=1$ is not imposed and $m(\emptyset) \neq 0$ can exist. This can introduce the notion of open world while assuming that the belief cannot be attributed to a subset of $\Omega$. In this case, $\emptyset$ can be interpreted as a proposal which is not in the frame of discernment $\Omega$ and that it is likely to be the solution to the problem as opposed to the closed world where the set $\Omega$ is assumed to be exhaustive.

A basic belief assignment is said to be simple (the acronym $S B B A$ can be found in literature) if it has two focal elements including $\Omega$. From the mass function, other belief functions such as plausibility (noted $p l$ ), credibility (bel), implicability $(b)$ and communality $(q)$ functions can be defined. These functions are dual measures and represent the same information expressed in different ways. Moreover, they can be translated from one to the other thanks to the Möbius transform [8], as follows between the mass function and the communality function:

$$
q(B)=\sum_{A \supseteq B} m(A) \quad \forall B \subseteq \Omega
$$

Modeling as a function of belief has no generic solution and, generally, it depends on the application [9, 10]. Based on Shafer's work [3] on simple basic belief assignments $(S B B A)$, Smets proposed the notion of generalized simple $B B A$ $(G S B B A)$ [11]. A $G S B B A$ can be noted $A^{\boldsymbol{\omega}(A)}$ and Smets showed that these weights $\boldsymbol{\omega}(A)$ for all $A \in 2^{\Omega} \backslash\{\Omega\}$ can be obtained by the following formula:

$$
\boldsymbol{\omega}(A)=\prod_{B \supseteq A} q(B)^{(-1)^{|B|-|A|+1}}
$$

\footnotetext{
${ }^{1}$ The term BBA for basic belief assignment is also found in literature.
} 
So, a generalized simple BBA can be defined as a function $\mu$ from $2^{\Omega}$ to $\mathbb{R}$ :

$$
\begin{aligned}
& \mu(A)=1-\boldsymbol{\omega}(A), \\
& \mu(\Omega)=\boldsymbol{\omega}(A), \\
& \mu(B)=O \quad \forall B \in 2^{\Omega} \backslash\{A, \Omega\}
\end{aligned}
$$

for each $A \neq \Omega$ and $\boldsymbol{\omega}(A) \in[0,+\infty[$. The function $\boldsymbol{\omega}$ is yet another representation of a non-dogmatic mass function and is called the conjunctive weight function. Many operations from weight functions and the meaning of these functions are not presented in this article, we invite the reader to refer $[12,11]$ for more details.

\subsubsection{Refinements/Coarsenings and Vacuous extension}

When applying the TBM to a real-world application, the determination of the frame of discernment $\Omega$, which defines the set of states on which beliefs will be expressed, is a crucial step. As noticed by Shafer [3, chapter 6], the degree of granularity of $\Omega$ is always, to some extent, a matter of convention, as any element of $\Omega$ representing a given state can always be split into several alternatives. Hence, it is fundamental to examine how a belief function defined on a frame may be expressed in a finer or, conversely, in a coarser frame. The concepts of refinement and coarsening can be defined as follows.

Let $\Theta$ and $\Omega$ denote two frames of discernment. A mapping $\rho: 2^{\Theta} \rightarrow 2^{\Omega}$ is called a refining of $\Theta$ (Figure 1) if it verifies the following properties:

1. The set $\{\rho(\{\theta\}), \theta \in \Theta\} \subseteq 2^{\Omega}$ is a partition of $\Omega$, and

2. For all $A \subseteq \Theta$ :

$$
\rho(A)=\bigcup_{\theta \in A} \rho(\{\theta\})
$$

$\Theta$ is then called a coarsening of $\Omega$, and $\Omega$ is called a refinement of $\Theta$.

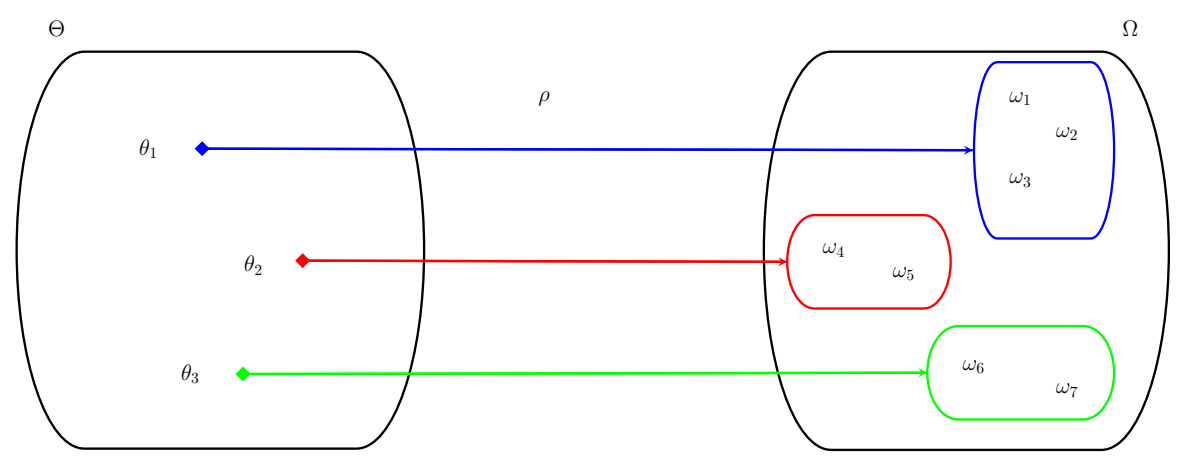

Figure 1: Illustration of a coarsening $\Theta$ of $\Omega$ associated with a refining $\rho$ of $\Theta$.

With this notion of refining, we can define the vacuous extension. This operation allows one to convey a mass function $m^{\Theta}$, expressing a state of belief on $\Theta$, to a finer frame $\Omega$, a refinement of $\Theta$. Stemming from the least committed 
principle [13], this operation is denoted with an arrow pointing up, and is defined by:

$$
m^{\Theta \uparrow \Omega}(\rho(A))=m^{\Theta}(A), \quad \forall A \subseteq \Theta,
$$

where $\rho$ is the refining of $\Theta$ in $\Omega$.

\subsubsection{Discounting}

When the resulting information in the belief function is not totally reliable, it may be necessary to discount this belief. In order to do that, a coefficient $\alpha$ can be used, which represents the knowledge of the source reliability and allows to redistribute the beliefs to the set $\Omega$ proportionally to the source reliability. The discounted belief function $m^{\alpha}$ can be deduced from $m$ and $\alpha$ (i.e. $m^{\alpha}=$ $\operatorname{Disc}(m, \alpha))$ and can be obtained by the following expression:

$$
\left\{\begin{array}{l}
m^{\alpha}(A)=\alpha m(A) \\
m^{\alpha}(\Omega)=1-\alpha+\alpha m(\Omega) .
\end{array}\right.
$$

In literature several methods have been developed to compute the discounting factor, e.g. [14]. A discounting factor can be defined in terms of sustainability information. This principle, called the principle of memory decay, has been presented by Philippe Smets in [15]. This principle states that each $B B A$ is discounted with time: the longer the time since the $B B A$ has been collected, the stronger the discounting. The $B B A$ is discounted by the reliability factor $\alpha(t)$ that is a decreasing function of time with $\alpha(0)=1$ and $\lim _{t \rightarrow+\infty} \alpha(t)=0$. Let a $B B A{ }^{t^{\prime}} m$ obtained at time $t^{\prime}$, a new $B B A{ }^{t^{\prime \prime}} m$ is computed at time $t^{\prime \prime}$ with $t^{\prime \prime}>t^{\prime}$ using the following formulation:

$$
\left\{\begin{array}{l}
t^{\prime \prime} m(A)=\alpha\left(t^{\prime \prime}-t^{\prime}\right) \times \quad t^{\prime} m(A) \\
t^{\prime \prime} m(\Omega)=1-\alpha\left(t^{\prime \prime}-t^{\prime}\right)+\alpha\left(t^{\prime \prime}-t^{\prime}\right) \times \quad t^{\prime} m(\Omega) .
\end{array}\right.
$$

In a general way, this discounting can be also formulate as following:

$$
t^{\prime \prime} m=\operatorname{Disc}\left(t^{\prime} m, \alpha\left(t^{\prime \prime}-t^{\prime}\right)\right) .
$$

\subsubsection{Combination rules}

When many sources share beliefs in relation to the validity of a hypothesis of $\Omega$, the different points of view can be fused using combination rules. Historically, within the framework of the belief function theory, the Dempster's conjunctive combination rule and the TBM conjunctive combination rule (also called the unnormalized conjunctive combination rule) have played an important part, especially thanks to their axiomatic justifications [15]. The merge of two distinct sources $m_{1}$ and $m_{2}$ can be made using the TBM conjunctive rule of combination, denoted by $m_{1} @ m_{2}=m_{1} @ 2$. This rule is commutative and associative and is defined by:

$$
m_{1} \bigcap_{2}(C)=\sum_{A \cap B=C} m_{1}(A) \cdot m_{2}(B), \forall C \subseteq \Omega
$$


The conjunctive weight function representation also appears particularly interesting when it comes to combining BBAs using the conjunctive combination rule. Indeed, let $m_{1}$ and $m_{2}$ be two BBAs with weight function $\boldsymbol{\omega}_{\mathbf{1}}$ and $\boldsymbol{\omega}_{\mathbf{2}}$. We have:

$$
\begin{aligned}
m_{1} @ m_{2} & =\left(\underset{A \subset \Omega}{\cap} A^{\boldsymbol{\omega}_{1}(A)}\right) \circledast\left(\underset{A \subset \Omega}{\cap} A^{\boldsymbol{\omega}_{\mathbf{2}}(A)}\right) \\
& =\bigoplus_{A \subset \Omega} A^{\boldsymbol{\omega}_{1}(A) \boldsymbol{\omega}_{\mathbf{2}}(A)}
\end{aligned}
$$

Due to undesirable behaviors (e.g. too important conflict $m(\emptyset)$ ) after the combination or need of source independence), and based on the works on SBBA and GSBBA, Denœux proposed in [12] a new rule of combination, the cautious rule. So, the combination of $m_{1}$ and $m_{2}$, two non dogmatic BBAs, using the cautious rule depends on the weight functions:

$$
\boldsymbol{\omega}_{1} \bigcap_{2}(A)=\boldsymbol{\omega}_{1}(A) \wedge \boldsymbol{\omega}_{2}(A), \forall A \in 2^{\Omega} \backslash \Omega,
$$

and the final combination $m_{1} @ m_{2}=m_{1} \bigotimes_{2}$ is obtained by:

$$
m_{1} \bigcap_{2}(A)=\bigcap_{A \subset \Omega} A^{\boldsymbol{\omega}_{1}(A) \wedge \boldsymbol{\omega}_{2}(A)} .
$$

where $\wedge$ denotes the minimum operator. Like the above rules, the cautious rule is commutative and associative. But the interested property is the idempotent property $(m @ m=m)$ which allows to combine two non distinct mass functions given by two non independent sources. Other combination rules have been proposed, e.g. Yager's combination [16] or Dubois and Prade's rules [17]. For more details about combination rules, the interested reader may be refer to [15, $18,19]$.

\subsubsection{Decision making}

Many solutions have been proposed to make decisions in the evidential framework (e.g. maximize the credibility or minimize the plausibility degrees), we present in this paper the retained solution for our application, the pignistic probability of Philippe Smets, defended within the transferable belief model [4], which is defined by the following equation:

$$
\forall \omega_{n} \in \Omega \quad \operatorname{Bet} P\left(\omega_{n}\right)=\frac{1}{1-m(\emptyset)} \sum_{A \ni \omega_{n}} \frac{m(A)}{|A|}
$$

where $|A|$ represents the cardinality of $A \subseteq \Omega$. Once the pignistic probability obtained, it is possible to use classic tools of statistical decision theory. Readers could find justifications and details of this transformation in [20].

\subsection{Multi-agent systems}

The ever more increasing needs to understand real and complex systems have encouraged modelers to implement modeling paradigms from distributed artificial intelligence $(D A I)$. Especially, the multi-agent paradigm has growed in 
the modeling of living systems. As in the previous section, essential concepts of multi-agent systems are presented and the interested reader may refer to $[1,21]$ for more details.

The term "agent" is a generic term for which no definition really comes to a consensus. A general definition ${ }^{2}$ comes from [1]. Ferber defines the agent as an autonomous virtual (or physical) entity that:

- can act in an environment,

- can perceive its environment,

- can communicate directly (e.g. by sending messages) or indirectly (e.g. via the environment) with other agents,

- is governed by a set of trends (e.g. objective optimization) and is limited by a set of constraints (e.g. limited resources),

- has competences and offers services.

From this definition, a definition of multi-agent systems $(M A S)$ can be given. It is a system composed of:

- an environment $E n$ with a certain metric,

- a set of objects $O b$ situated in $E n$,

- a set of agents $A g=\left\{a g_{1}, \ldots, a g_{i}\right\}$ that can perceive and manipulate the objects,

- a relation set $R_{O b}$ between the objects,

- a relation set $R_{A g}$ between the agents (e.g. communicate, share resources etc.),

- a relation set $R$ between the agents and the objects (e.g. carry, move etc.),

Historically, two types of agent can be distinguished in MAS: reactive or cognitive agents. First ones have no explicit environment representation and they react reflexively to stimuli (e.g. an agent modelizing an insect). Cognitive agents have a more developed environment representation, explicit goals, memory abilities or capabilities of individual reasoning. There may also be hybrid agents and multi-agent systems composed of reactive and cognitive agents, as in our application presented thereafter.

\footnotetext{
${ }^{2}$ A simplified version of Ferber's definition is presented on purpose.
} 


\subsection{Validation and calibration of multi-agent simulations and associated prob- lems}

Validation of any model is an important task [2]. This problem matters of course within the framework of multi-agent simulations, especially with their expanding importance and their implementations in various fields [22]. Validate a MAS usually requires expert interventions, expert typically compares the real system outputs to their modeled equivalents. Comparing the model to reality is done using various tests, that can be objective, quantitative, subjective or qualitative. Because the information conveyed in such systems are generally numerous, very heterogeneous and largely inside the agents themselves, the validation of multi-agent simulations is directly done through observation of agents (and/or their communications [23]). Observation of emergent properties is more difficult, particularly because of the difficult characterization of such properties.

Automation of observation process has already been addressed and architectures (e.g. [24]) have been presented to validate agent based simulations. In a recent publication [25], we have also shown that a statistical approach of observation could be interesting. The sample survey theory [26] seems to provide a non-negligible interest if the number of agents is too high (several thousands).

But the real system complexity inevitably leads to a difficult access to parameter values, e.g. it seems rather difficult to observe and know status of each ant in a colony at a given time. These difficulties are all the more important as the system dynamics lead to rapid, regular, agent-specific changes of these parameters. Real parameters can only be observed occasionally and usually at so called "simple" or "obvious" moments: at the initialization step, during downturns (e.g. when ants enter in diapause stage) or at the end of the experiment. Within the framework of the agent-based simulation validation, the lack of knowledge of these values has a direct impact on possible times of validation: it seems intuitively difficult to validate a model at a time $t_{v}$ when the known values of the real system have been observed at a time $t_{w} \neq t_{v}$.

However, when the observation data are only available at $t_{w}$, and just because the simulation costs are important, it may be interesting to estimate the model state and predict the validation results. If the parameter dynamics can be known or estimated and that the imperfections can be managed, even if the system is complex, it seems that predictions may be possible. The usual definition of validation (i.e. "compare the results of the model - in our case, outputs of the simulation - to those of the real system") can be extended into "check if the model is still in agreement with the real system". Thus, it seems interesting to develop a validation system based on recent work, the previous remarks, and based on the belief function theory to manage imperfect information.

Moreover, on an other hand, let the simulation be initialized with a specific value of $\Omega$. $\Omega$ can take $K$ values in the hypothesis set $\Omega=\left\{\omega_{1}, \omega_{2}, \ldots, \omega_{K}\right\}$, and outputs and behavior of the model vary depending on the values taken by $\Omega$. It seems interesting to browse all - or some - of these values of $\Omega$ and iteratively validate the simulation conditionning to the selected value taken by 
$\Omega$. So, the validation is a part of an iterative process to successively retroactive on simulation inputs, in order to obtain a model enough closed to the reality: this iterative process is called calibration [2]. The pyramidal validation system presented hereafter is integrated into a more general framework of calibration that consists in choosing the best value of $\Omega$. In practice, the calibration process is based on an inductive heuristic, presented in [27]. We present afterward, the simulation validation process and the appropriate agent based architecture.

\section{Validation of multi-agent simulations based on the belief function theory}

In this section, the proposed validation architecture is detailed: the subsection 3.1 presents the general multi-agent organisation of validation system and the next one presents the interest of belief functions within such a framework.

\subsection{Pyramidal architecture}

We consider a real system and its multi-agent model, both evolving in time $t \in\left[t_{0} ; t_{o b s}\right]$. An expert can give us the set of validity domains Dom = $\left\{\operatorname{dom}_{1}, \ldots, \operatorname{dom}_{x}, \ldots, d o m_{X}\right\}$ of the parameters $P=\left\{p_{1}, \ldots, p_{x}, \ldots, p_{X}\right\}$ observed at the time $t_{o b s}$. These parameters concern properties of agents and groups of agents.

The adopted validation strategy is to "agentify" each parameter of $P$. So, we consider the time $t_{v} \in\left[t^{-} ; t^{+}\right]$with $\left[t^{-} ; t^{+}\right] \subseteq\left[t_{0} ; t_{o b s}\right]$. At $t_{v}$, each $p_{x}$ is a source that can answer the next question:

$Q$ : "At $t_{v}$ and considering $\omega_{k}$, am I still in agreement with facts found by the expert at $t_{o b s}$ ?".

Each parameter can answer the question $Q$ whenever wanted between the minimum time constraint $t^{-}$and the maximum time constraint $t^{+}$. To answer the question $Q, p_{x}$ is able to probe the set of appropriate agents $A g^{\prime} \subseteq A g$ by transferring them the related question $Q$. The answer of the question $Q$ belongs to the set $\Omega_{k}=\{y e s, n o\}$.

As in most multi-agent simulation platforms, simulation could be controlled (i.e. launching, agent creation...) by an agent named Launcher-Agent ${ }^{4}$. In these platforms, this agent usually initializes the simulation and launches the simulation agents. In the proposed architecture, the Launcher-Agent also launches the Validation-Agent needed to validate simulations and responsible for the set of Parameter-Agents $P$ ( $c f$. figure 2). So, a validation system on two intermediate

\footnotetext{
3"Agentify" is an expression used to turn system actors or non-actors (e.g. the parameters) into an agent in the simulated model.

${ }^{4}$ Name used for example in the MadKit platform [28].
} 


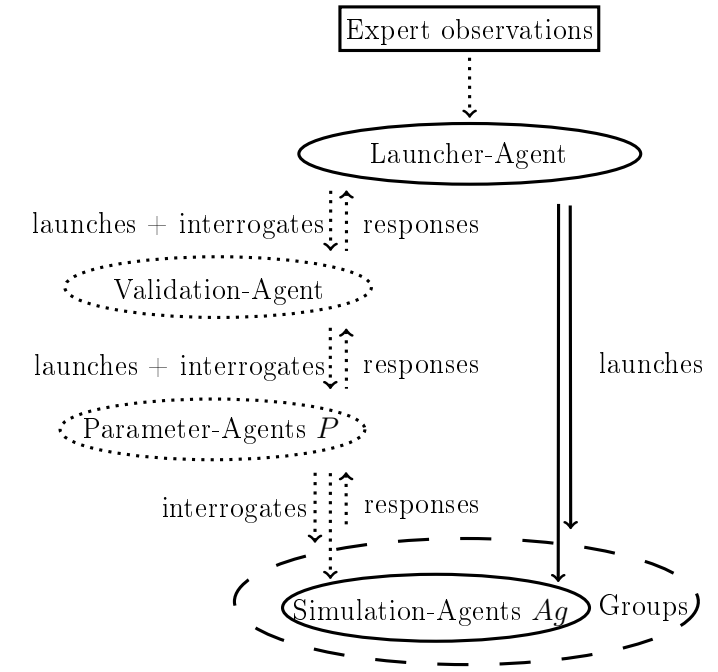

Figure 2: Pyramidal architecture of validation strategy.

levels, between the Launcher-Agent and simulation agents $A g$, has been developed. This architecture is called pyramidal because of the increasing number of involved agents from the Validation-Agent level to the last level.

\subsubsection{General behaviour of validation agents}

Whatever the validation level (Validation-Agent or Parameter-Agents), an agent has always the same general behaviour: it receives a question, it transfers this question to appropriate "subordinate" agents and waits for responses before informing its superior agent ( $c f$. figure 3 ).

The first validation level, the Validation-Agent manages the validation process (e.g. start, stop, time management...) after being created by the LauncherAgent. Once launched, this agent launches Parameter-Agents (their number varies with expert observations). At a minimum time constraint $t^{-}$, it informs each Parameter-Agent of:

1 - information of all the potential simulation agents $A g$ capable of answering the question $Q$,

2 - the validity domain $d o m_{x}$ of the parameter $p_{x}$ at $t_{o b s}$,

3 - the next time limit $t^{+}$before which the Parameter-Agents should have answered.

As shown on figure 3, once launched, a Parameter-Agent $p_{x}$ :

1 - selects an agent group $A g^{\prime} \subseteq A g$ to probe,

2 - informs the $A g^{\prime}$-agents of the question $Q$ and the validity domain $d_{0 m}$ of the parameter $p_{x}$ at $t_{o b s}$, 


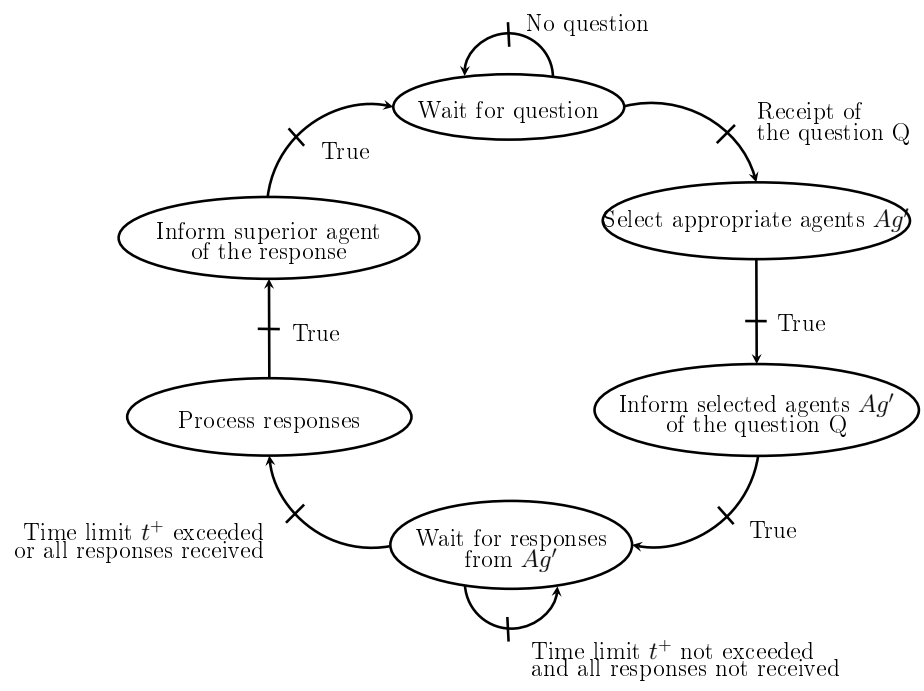

Figure 3: General behaviour of a specific Parameter-agent $p_{x}$

3 - communicates the time limit $t^{+}$given by the Validation-Agent.

\subsubsection{Behaviour of the probed simulation agents}

At each simulation step, before running its regular life cycle, a simulation agent $a g_{i}$ verifies if it receives a message $Q$ from a Parameter-Agent $p_{x}$. When it is contacted at $t^{-}$, it checks if it is concerned by this request. If so, it saves the question $Q$, the time constraint $t^{+}$and the parameter validity domain $d o m_{x}$. Depending on its activity, it can answer the question between the time steps $t^{-}$and $t^{+}$. At $t_{v} \in\left[t^{-} ; t^{+}\right]$, to answer the question, $a g_{i}$ takes into account the validity domain $\operatorname{dom}_{x}$ and compares it to the current value of $p_{x}$ and send its response to its $p_{x}$.

\subsection{Interest of belief functions to validate multi-agent simulations}

To handle imperfect information exchanged by agents, responses are expressed as belief functions. We present in this section the way of construction and management of basic belief assignments in the validation system. An illustrative example is presented in figure 5 .

\subsubsection{Evidential response of the simulation agents $\mathrm{Ag}$}

At $t_{v} \in\left[t^{-} ; t^{+}\right]$, to answer the question, $a g_{i} \in A g^{\prime}$ (with $A g^{\prime} \subseteq A g$ ) takes into account the validity domain $d_{0} m_{x}$ and compares it to the current value of $p_{x}$. It sends an evidential response to its $p_{x}$. Various methods can be implemented 


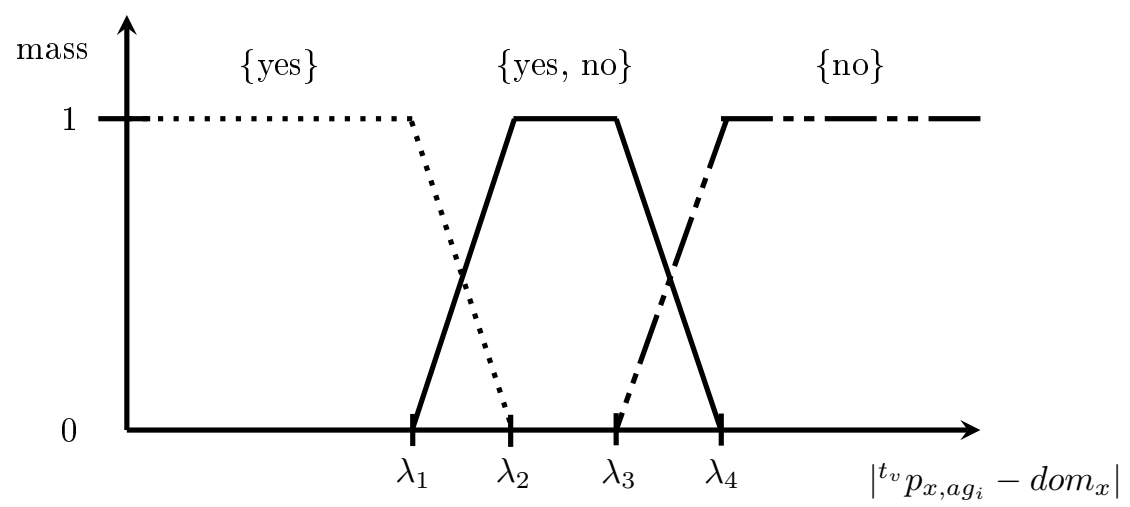

Figure 4: Method of mass assignment for a simulation agents $a g_{i}$ and for a quantitative parameter $p_{x}$. The value ${ }^{t_{v}} p_{x, a g_{i}}$ corresponds to the value of the $p_{x}$-parameter of the agent $a g_{i}$ at the time $t_{v}$ and $d o m_{x}$ is the limit value observed at $t_{o b s}$. The expression $\left|{ }^{t} p_{x, a g_{i}}-d o m_{x}\right|$ is an absolute value and the values $\lambda_{1}, \lambda_{2}, \lambda_{3}$ and $\lambda_{4}$ correspond to thresholds currently defined empirically. So, for example, when the result of the expression $\left|{ }^{t} p_{x, a g_{i}}-d o m_{x}\right|$ is inferior to $\lambda_{1}$, the value ${ }^{t_{v}} p_{x, a g_{i}}$ seems enough similar to the limit $d o m_{x}$ to be considered as a correct value and to assign all the belief to the singleton yes.

to create the mass function ${ }^{t_{v}} m_{x, a g_{i}}^{\Omega_{k}}: 2^{\Omega_{k}} \mapsto[0,1]$, where $\Omega_{k}=\{y e s, n o\}$. For example, in the case of a quantitative parameter within the framework of our application (see section 5), we have defined the mass assignment method presented on figure 4 .

\subsubsection{Management of basic belief assignments by the Parameter-Agents}

Between its own time limits $\left[t^{-} ; t^{+}\right]$, a Parameter-Agent $p_{x}$ can receive multiple responses at different times $t_{v}$ from subordinate $A g^{\prime}$-agents. To get a $B B A$ related to the parameter validity, $p_{x}$ has to combine the mass functions ${ }^{t_{v}} m_{x, a g_{i}}^{\Omega_{k}}$ but this can only be done at the time $t^{+}$(i.e. when all the Simulation-Agents should have answered the question).

So, before combining the $B B A s, p_{x}$ discounts them by respecting the principle of memory decay described in sub-section 2.1.3. The $B B A^{t_{v}} m_{x, a g_{i}}^{\Omega_{k}}$ is discounted by the reliability factor $\alpha\left(t^{+}-t_{v}\right)$ with $t_{v} \in\left[t^{-} ; t^{+}\right]$. The new $B B A^{t^{+}} m_{x, a g_{i}}^{\Omega_{k}}$ representing the belief at time $t^{+}$can be obtained with the next expression:

$$
{ }^{t^{+}} m_{x, a g_{i}}^{\Omega_{k}}=\operatorname{Disc}\left({ }^{t_{v}} m_{x, a g_{i}}^{\Omega_{k}}, \alpha\left(t^{+}-t_{v}\right)\right), \quad \forall a g_{i} \in A g^{\prime}
$$

Finally, to get the $B B A{ }^{t^{+}} m_{x}^{\Omega_{k}}$ related to the validity of the parameter $p_{x}$ at $t^{+}$, the Parameter-Agent $p_{x}$ combines all the ${ }^{t^{+}} m_{x, a g_{i}}^{\Omega_{k}}$ with the cautious rule of Denœux ( $c f$. sub-section 2.1.4):

$$
{ }^{t^{+}} m_{x}^{\Omega_{k}}=\bigotimes_{a g_{i} \in A g^{\prime}} \quad{ }^{t^{+}} m_{x, a g_{i}}^{\Omega_{k}}
$$




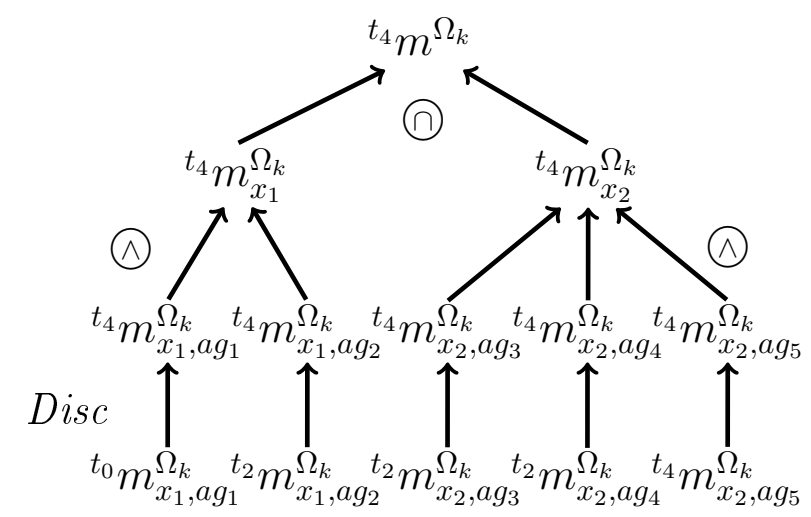

Figure 5: Example of a general architecture of belief functions with five Simulation-Agents, two parameters and between the time limits $t_{0}$ and $t_{4}$.

In this context, the cautious rule of combination is preferred for its idempotence property since the surveyed agents of $A g^{\prime}$ may not be completely independent (e.g. they can interact together, evolve with the same behaviour models...).

\subsubsection{Evidential validation at the Validation-Agent level}

The last validation level is to combine the $B B A s{ }^{t^{+}} m_{x}^{\Omega_{k}}$ given by the ParameterAgents of $P$ at $t^{+}$. Currently, because the Parameter-Agents probe different Simulation-Agents and attempt to estimate the validity of different parameters, they can be considered totally independent. The conjunctive rule of combination seems to be well adapted. So, the final basic belief assignment $t^{+} m^{\Omega_{k}}$ can be obtained with the next equation:

$$
{ }^{+} m^{\Omega_{k}}=\bigoplus_{p_{x} \in P} t^{+} m_{x}^{\Omega_{k}}
$$

Once this last $B B A{ }^{t^{+}} m^{\Omega_{k}}$ obtained, it only remains to forward the information to its Launcher-Agent. This Launcher-Agent can be able to compute the BBA at time $t_{o b s}$. The following discounting operation is realized to obtain this BBA:

$$
{ }^{t_{o b s}} m^{\Omega_{k}}=\operatorname{Disc}\left({ }^{+} m^{\Omega_{k}}, \alpha\left(t_{o b s}-t^{+}\right)\right) .
$$

\subsection{End of the simulation}

In some cases, the functions or dynamics, describing the system, are monotonic. Thus, when the belief of proposition $\{n o\}$ exceeds a threshold $\Delta$, the validation step is aborted. This value $\Delta$ could be computed by a learning process or given by a human expert. If the threshold is not exceeded, the process 
can continue until the time $t^{+}=t_{\text {obs }}$. The final BBA representing the belief at time $t_{o b s}$ can be obtained by the next expression:

$$
m^{\Omega_{k}}={ }^{t_{o b s}} m^{\Omega_{k}} .
$$

This last sept of validation is summed up by the following algorithm 1 .

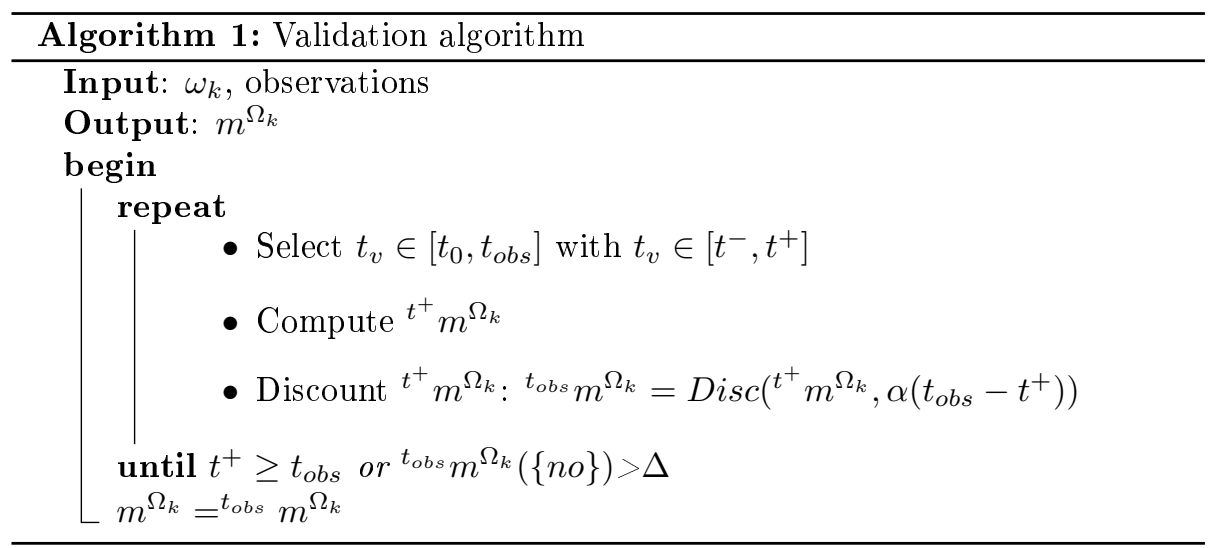

\section{Calibration}

In the previous section, the validation process was described. In this process, only one assumption $\omega_{k}$ is compared to the real data given by the expert. But in our case, the aim is to obtain a model enough closed to the reality. For that, two solutions could be used. The first one is to compare all the hypotheses to real data. This solution requires a prominent computation time when the number of assumption increases. The last solution, and one that will be selected, is to use a intelligent research strategy in order to find the testable hypotheses. This solution is based on a heuristic described in [27]. Thus if $K$ assumptions are tested, $K$ BBAs are computed in the validation phase. These BBAs are not defined on a common frame of discernment. Before to combine these BBAs, the operation of vacuous extension is used.

\subsection{Expressing pieces of information in a common frame: vacuous extension}

Following the validation phase, if $K$ assumptions $\omega_{k}$ with $k \in[1, K]$ have been tested then $K$ belief functions have been defined. Each function $m^{\Omega_{k}}$ represents the belief on the set of the possible answers (yes or no) to the question: " $\omega_{k}$ is it still in agreement with facts found by the expert ?" Frames $\Omega$ being refinement of $\Omega_{k}$, each information $m^{\Omega_{k}}$ can be expressed on $\Omega$ by a vacuous extension (8):

$$
m^{\Omega_{k} \uparrow \Omega}\left(\rho_{k}(A)\right)=m^{\Omega_{k}}(A), \quad \forall A \subseteq \Omega_{k},
$$

where $\rho_{k}$ is the refining of $\Omega_{k}$ on $\Omega$ illustrated in Figure 6 , and defined by $\rho_{k}(\{y e s\})=\{k\}$ and $\rho_{k}(\{n o\})=\overline{\{k\}}$. Thus, for all $k \in[1, K]$ : 


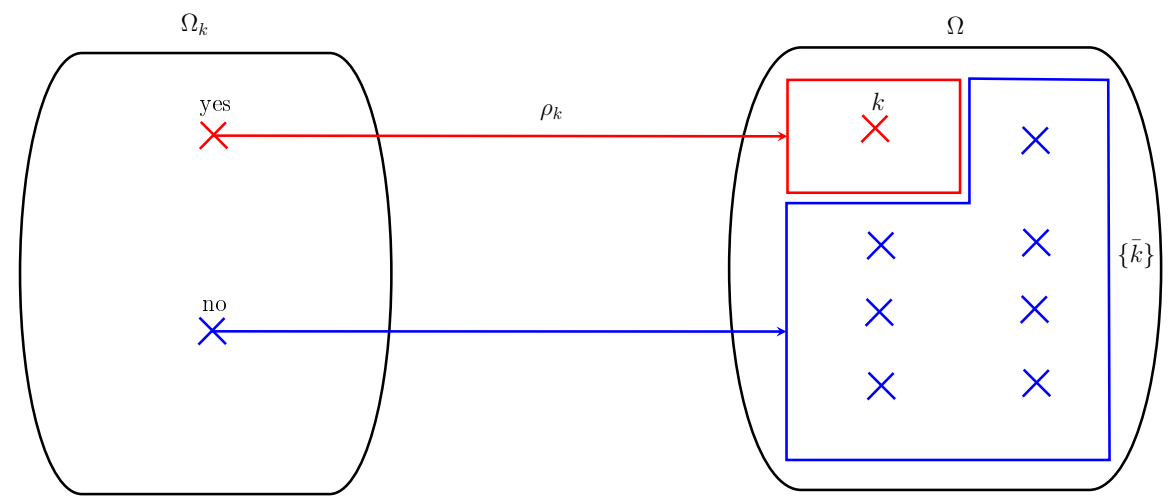

Figure 6: Refining $\rho_{k}$ allowing one to transport the information $m^{k}$ on $\Omega$.

$$
\begin{cases}m^{\Omega_{k} \uparrow \Omega}(\{k\}) & =m^{\Omega_{k}}(\{\text { yes }\}) \\ m^{\Omega_{k} \uparrow \Omega}(\overline{\{k\}}) & =m^{\Omega_{k}}(\{\text { no }\}) \\ m^{\Omega_{k} \uparrow \Omega}(\Omega) & =m^{\Omega_{k}}\left(\Omega_{k}\right)\end{cases}
$$

In the following, mass functions $m^{\Omega_{k} \uparrow \Omega}$ are denoted $m^{k, \Omega}$.

\subsection{Combination and decision}

Now, the BBAs are defined on a same frame of discernment and they can be combine with the conjunctive combination rule:

$$
m^{\Omega}=\underset{k=1, \cdots, K}{\bigcap} m^{k, \Omega}
$$

The steps of the whole calibration system are illustrated in figure 7 .

With the mass $m^{\Omega}$, the decision can be obtained using the maximum of pignistic probability (eq. 16).

\section{First results within the framework of the forensic entomology project}

During a criminal investigation, it is essential to obtain a maximum of information on the conditions of a manslaughter. Many methods to exploit the indications on the murder scene are known but, for large post-mortem intervals (PMI), only one of these techniques is useful in practice: forensic entomology. It consists in studying the insects found on a cadaver to estimate the time of its death. Modern PMI entomology estimation methods are based on insect development models but because of the important system complexity, results given by the experts are imperfect. To improve the decision-making and assist the forensic scientists, a decision support system (DSS) has been developed to consider all the ecosystemic parameters and a significant quantity of biological models (e.g. usually an expert can only use one or two single model). Therefore, 


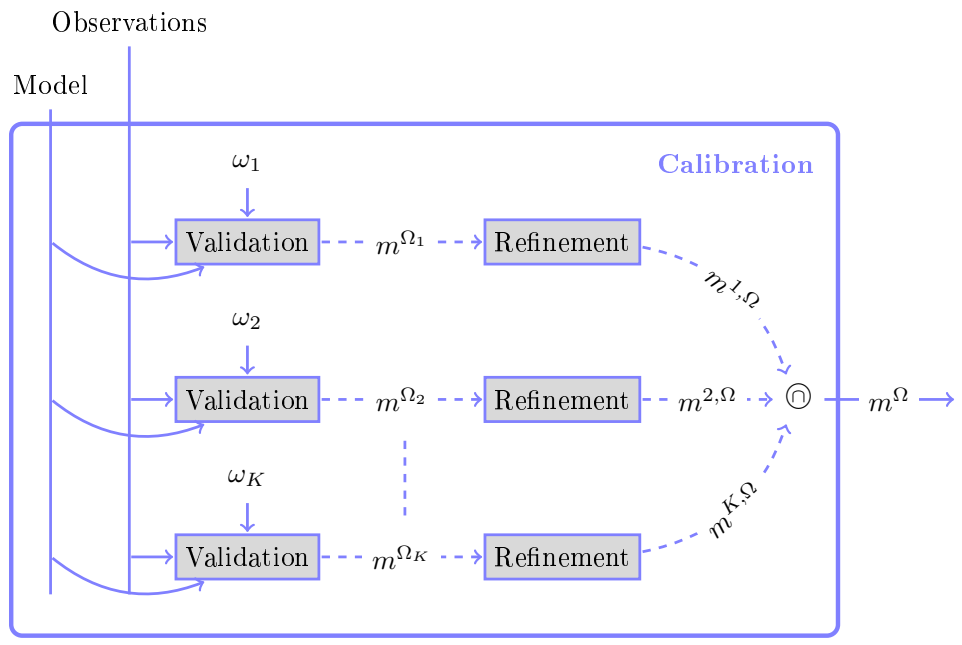

Figure 7: Presentation of the evidential calibration process, included the validation, refinement (eq. 23) and combination (eq. 24) steps.

the DSS is initialized with some specific entomology models found in literature, e.g. [29], to find the agreements between them. For this, the structure of the presented system is ameliorated: the principle described above (c.f., fig. 7) is duplicated. So, let $j$ be a model, with $j \in[1, J]$, then a BBA $m_{j}^{\Omega}$ can be computed. The $J$ mass functions $m_{j}^{\Omega}$, obtained by the $J$ models and considered as distinct and reliable, are combined using the conjunctive rule of combination. Let us denote $m$ the resulting mass function:

$$
m=\underset{j=1, \ldots, J}{\bigcap} m_{j}^{\Omega} .
$$

The general architecture of the DSS is presented in figure 8.

This project is based on a predictive multiagent model of insect development and cadaver decomposition in a complex ecosystem. It is used to determine if a hypothesis - a possible time of death - is coherent with the observations available on the ecosystem of the crime scene and the entomofauna found on the victim. The proposed pyramidal architecture has been implemented to compare the simulations to the reality given by experts at the cadaver discovery (e.g. which species, numbers of insects by species, reached development rates etc.). The validation system has been integrated into a recursive process of calibration to calibrate the system and detect the most probable time of death. More information about this model, the real system and the DSS can be found in [30].

The proposed method has been studied in a real case in which the person had disappeared around June 16th and the cadaver was found on June 


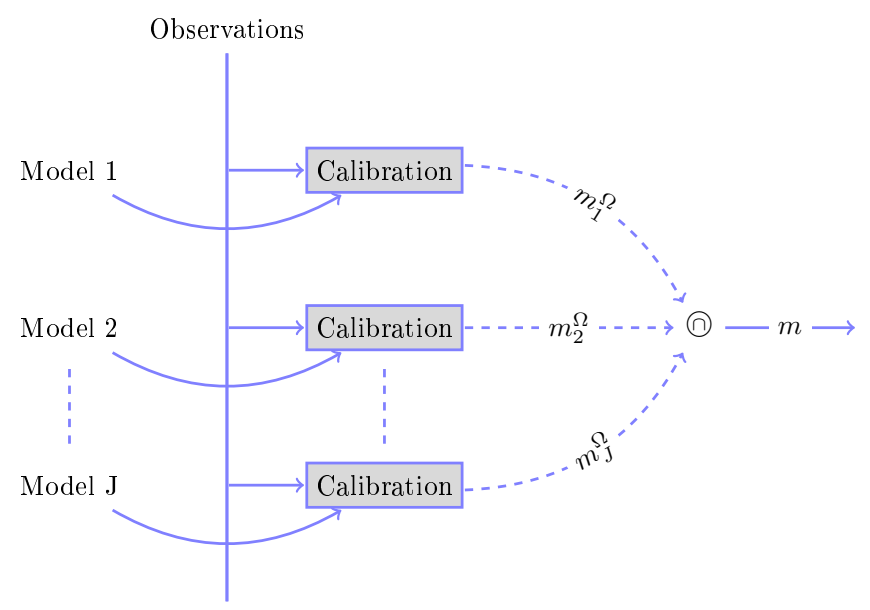

Figure 8: Illustration of the general architecture with J models.

$29 \mathrm{th}^{5}$. With this interval of search, we can define $\Omega$. In our case, an accuracy of one hour is sufficient for this application. So $\Omega$ is defined as follows: $\Omega=\left\{\omega_{1}, \ldots, \omega_{k}, \ldots, \omega_{313}\right\}$ where $\omega_{k}$ represents one possible moment (Day/Hour) of death. Experts have identified three species for which many development rates have been calculated or estimated (i.e. it can have different laying moments for the same species and so at the cadaver's discovery, insects of same species can have different development rates). Thus, ten entomological models are used to determine the time of death of a cadaver. In this example, the development rate parameters are agentified and have to answer question $Q$ :

$Q:$ "At $t_{v}$, am I still in agreement with the development rate given by the expert at $t_{\text {obs }}$ (the date of cadaver discovery)?".

A heuristic is used to select the hypotheses to be tested in the validation step. Thus, this example is only based on fifteen hypotheses. For these hypotheses, the development rates are estimated and the Parameter-Agents interview the Simulation-Agents of the concerned species to answer the question. In the figures 9 at 18, the basic belief assignment for each hypotheses selected is represented. It can be observed that is very difficult after this validation step to conclude about the time of death.

The figure 19 shows the pignistic probability obtained at the end of calibration step. By applying the maximum pignistic probability, the time of death is June 24 th at 22 o'clock. These first results are really interesting because ex-

\footnotetext{
${ }^{5}$ For confidentiality reasons, some information, e.g. the year and other details about crime circumstances, are omitted.
} 


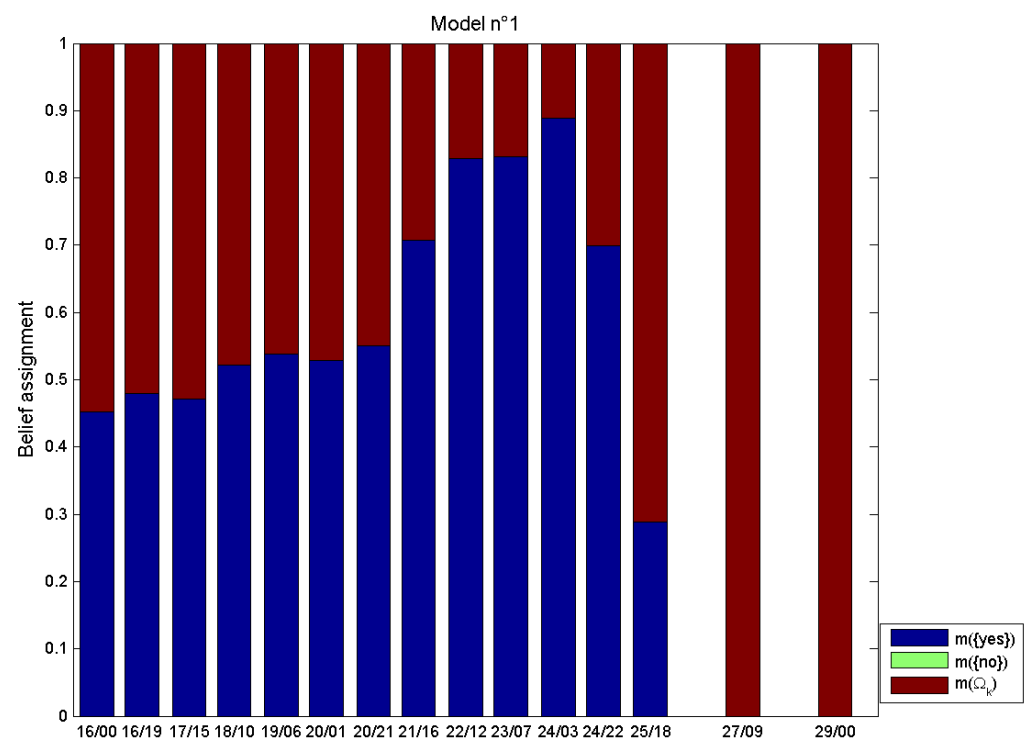

Figure 9: Basic belief assignment obtained with model $n^{\circ} 1$ after validation step.

perts had estimated the time of death around June 24th. The global system has still to be validated, but already, experts agree that the use of all ecosystemic information appears to be useful to estimate the time of death and make a more reliable and prudent decision.

\section{Discussions and conclusions}

The proposed validation architecture of agent based simulation allows to take into account imperfect data while being faster and more efficient than other validation methods usually used in multi-agent simulation platforms. With slight changes in these platforms, this pyramidal architecture can be easily integrated.

At the moment, only the validation of agent and group properties are developed and implemented but possible extensions on validations of the states of environment, objects and their different relations ( $c f$. section 2.2) could be proposed. Moreover, even if the quality of the results can be dependent on several characteristics, such as the assignment method of beliefs of Simulation-Agents, various methods of assignment have been proposed in literature. Only the reasoning from quantitative parameters has been implemented but later work will concern qualitative ones (e.g. reasoning on qualitative parameters could be very useful in our forensic application). For this, it is planned to switch to different works such as [31]. To go more thoroughly into the study of impacts on the fi- 


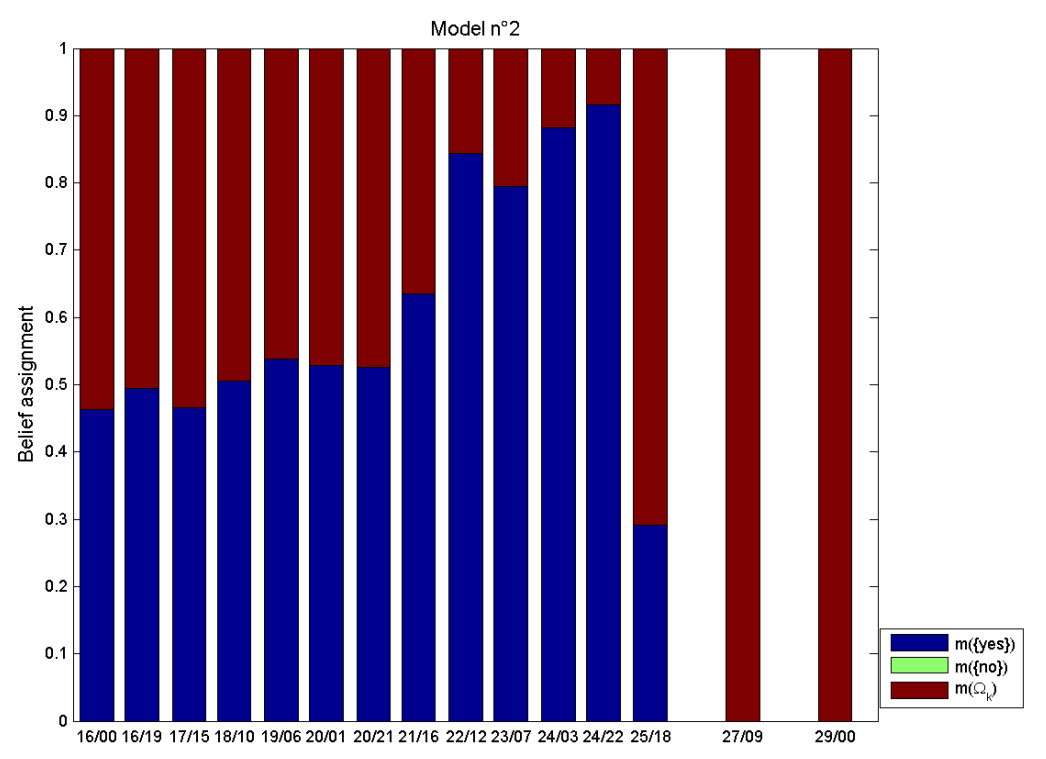

Figure 10: Basic belief assignment obtained with model $n^{\circ} 2$ after validation step.

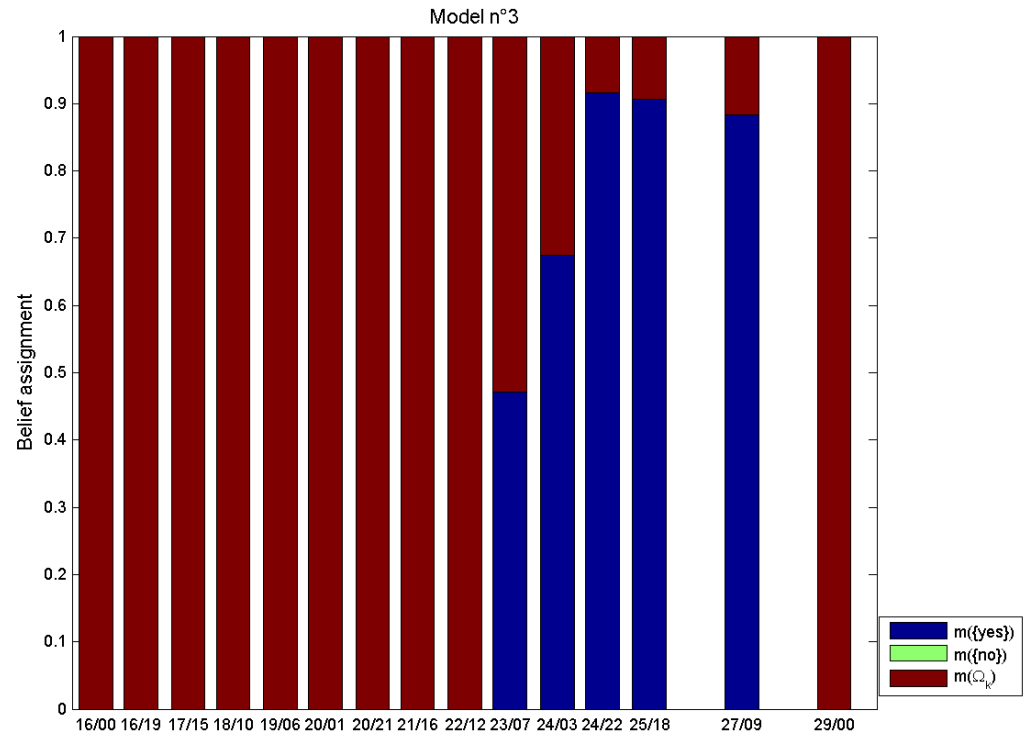

Figure 11: Basic belief assignment obtained with model $n^{\circ} 3$ after validation step. 


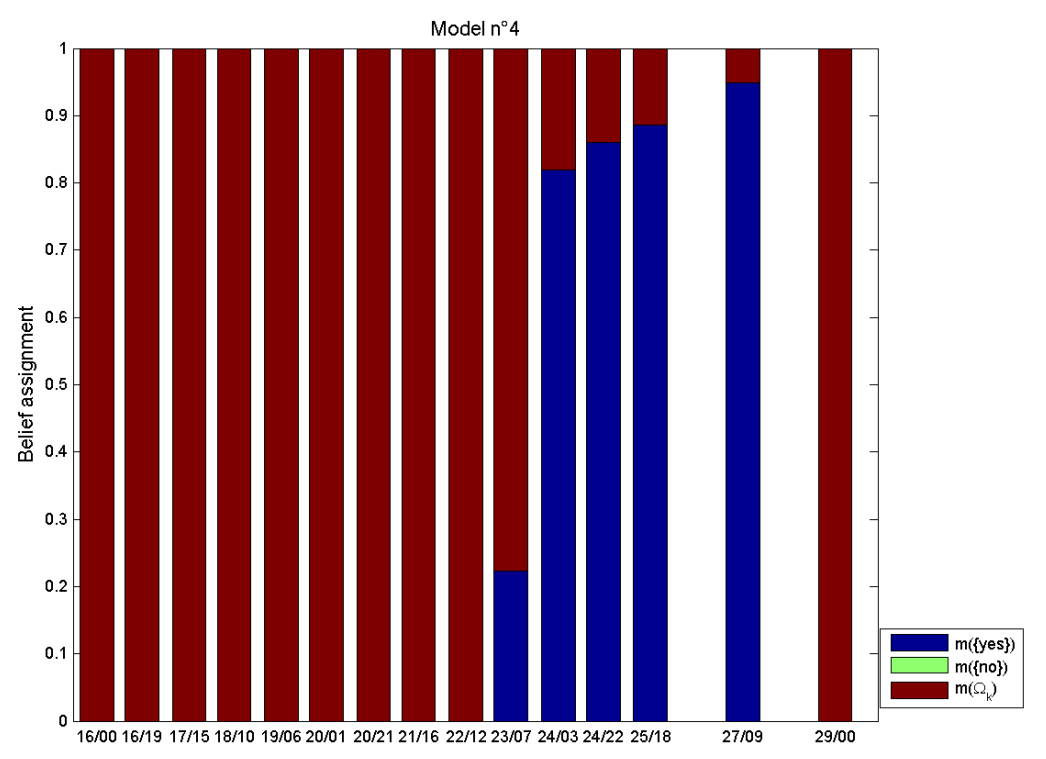

Figure 12: Basic belief assignment obtained with model $n^{\circ} 4$ after validation step.

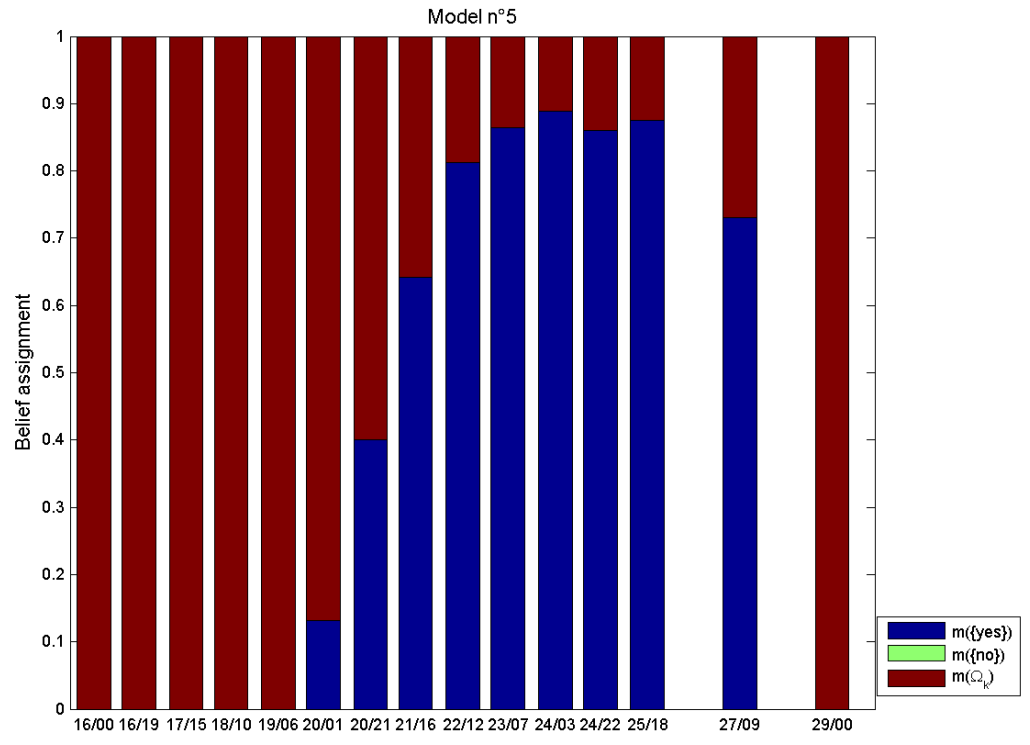

Figure 13: Basic belief assignment obtained with model $n^{\circ} 5$ after validation step. 


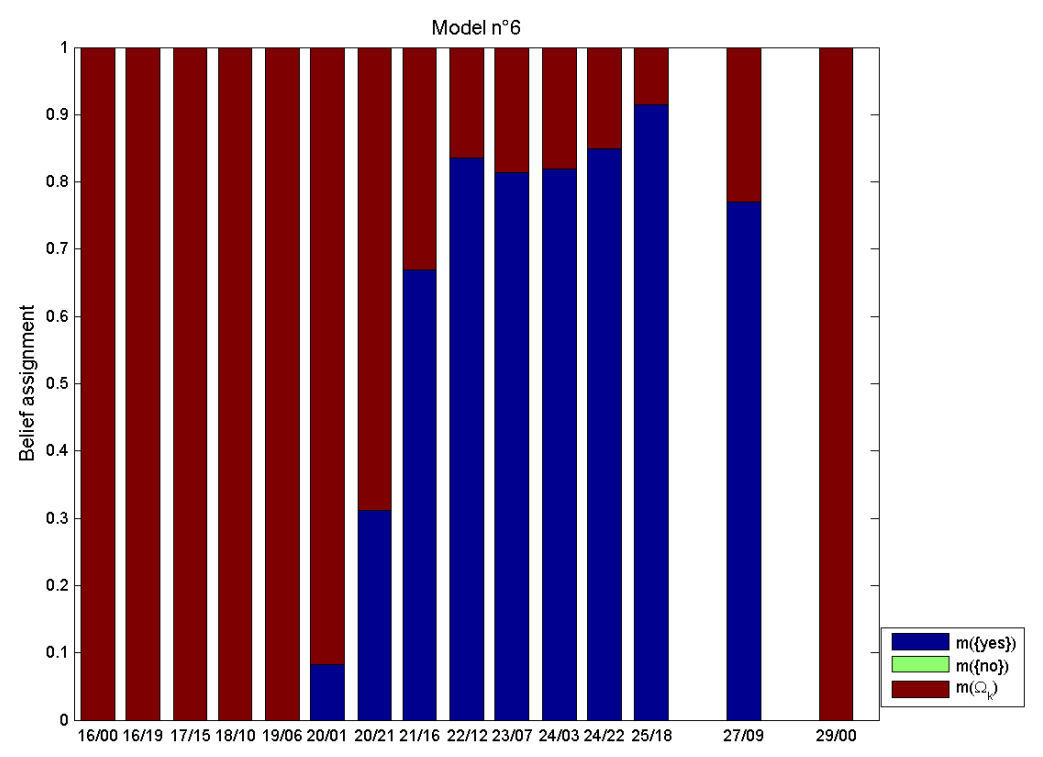

Figure 14: Basic belief assignment obtained with model $n^{\circ} 6$ after validation step.

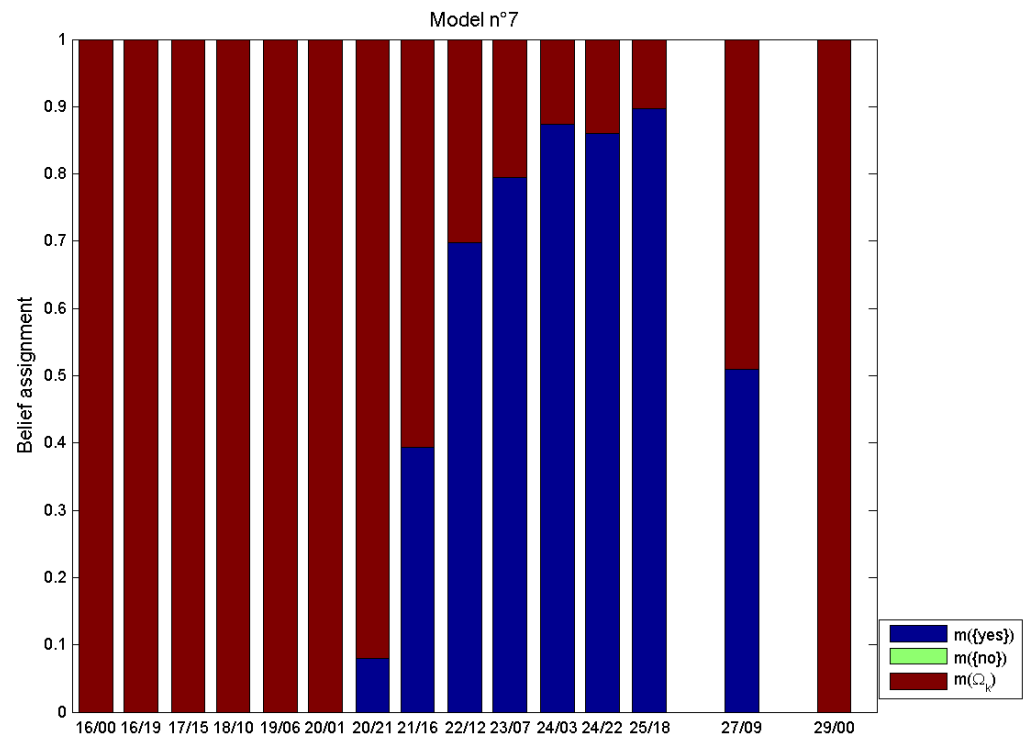

Figure 15: Basic belief assignment obtained with model $n^{\circ} 7$ after validation step. 


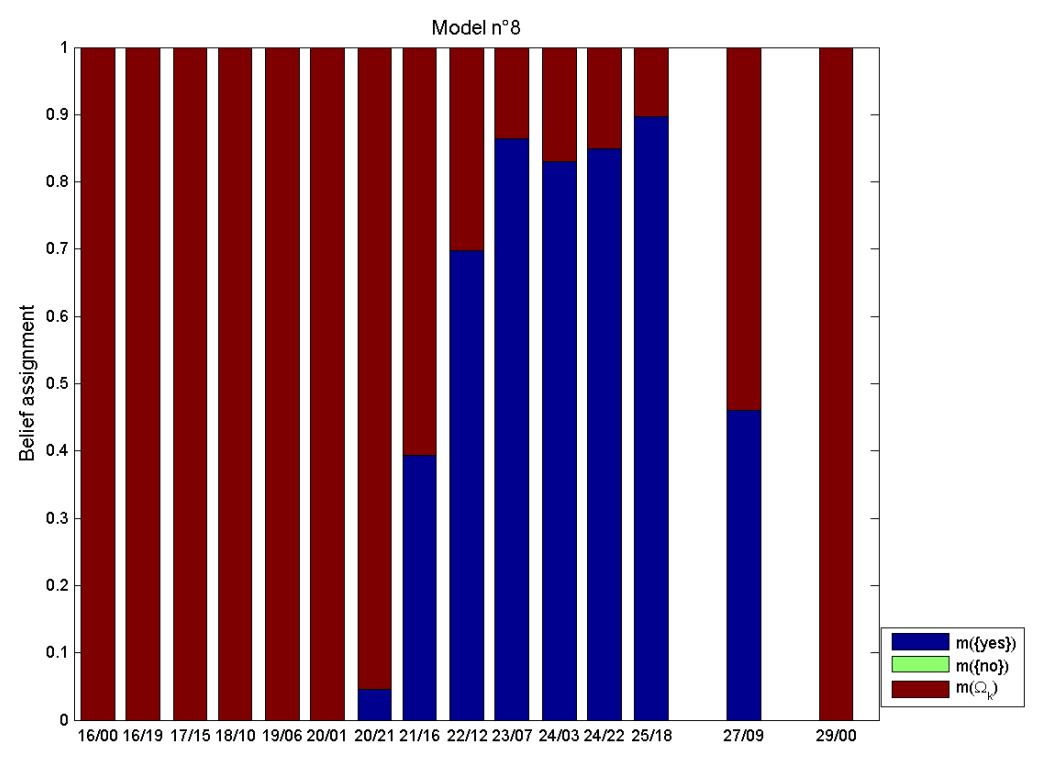

Figure 16: Basic belief assignment obtained with model $n^{\circ} 8$ after validation step.

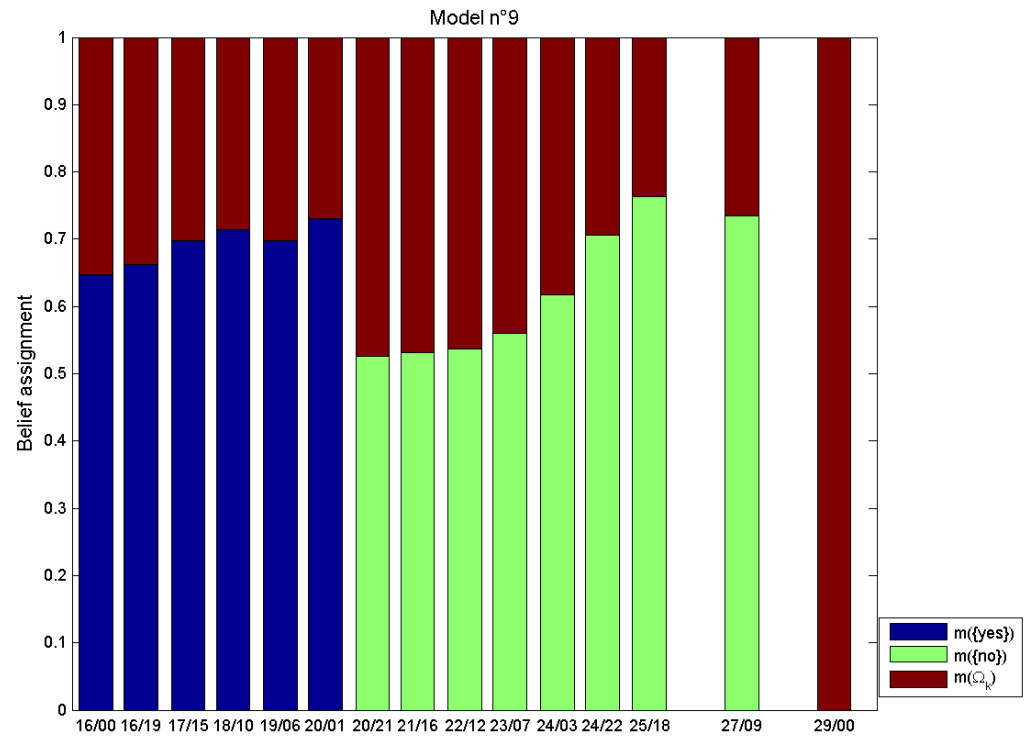

Figure 17: Basic belief assignment obtained with model $n^{\circ} 9$ after validation step. 


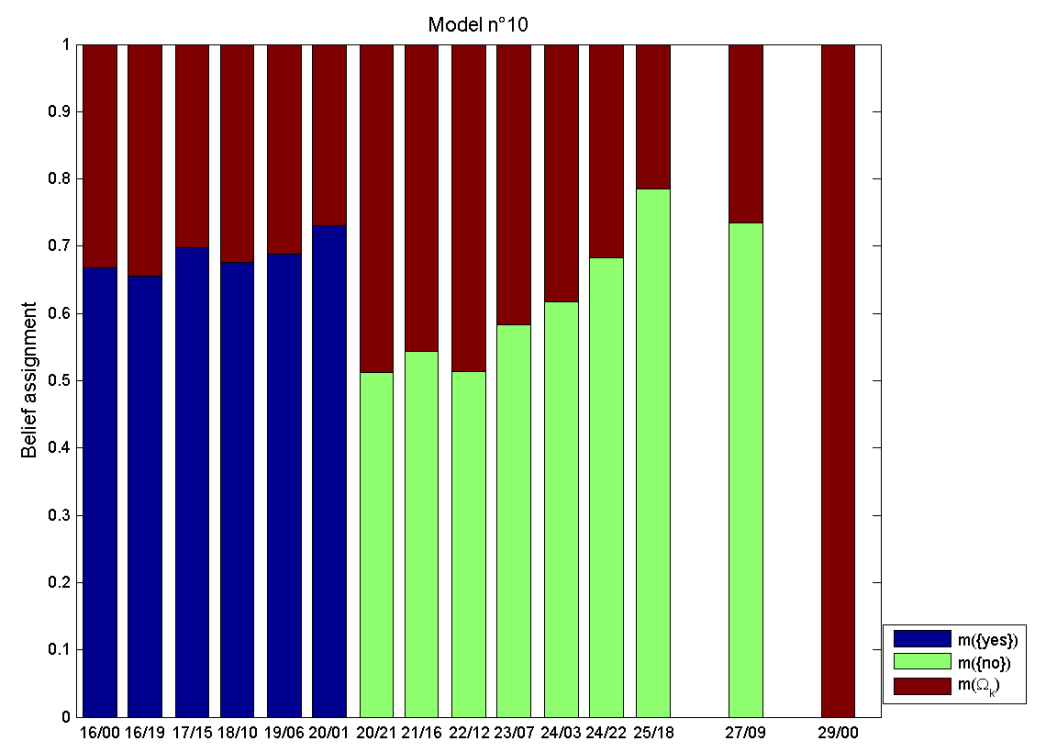

Figure 18: Basic belief assignment obtained with model $\mathrm{n}^{\circ} 10$ after validation step.

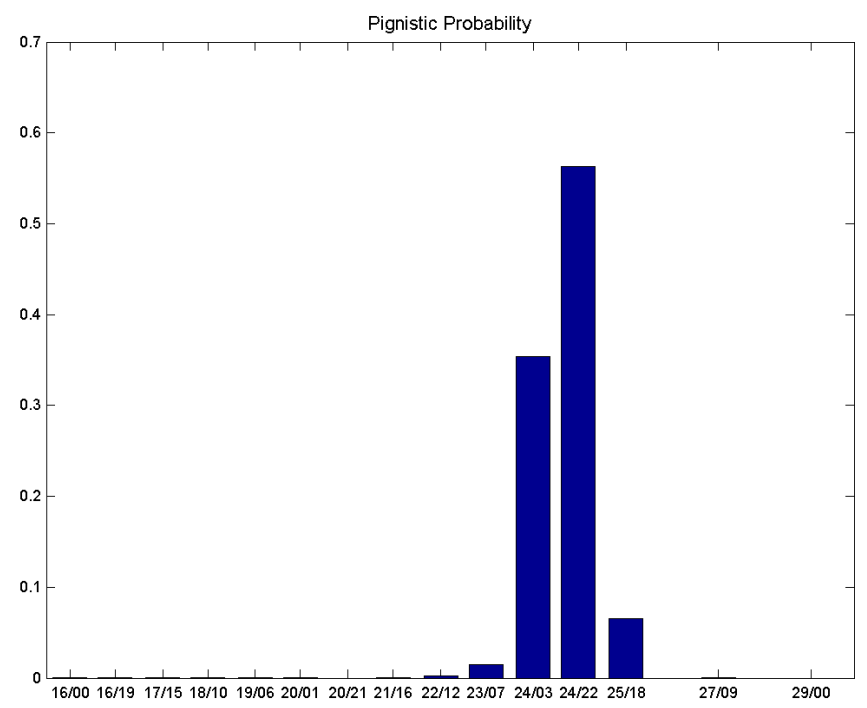

Figure 19: Final pignistic probability generated after using the DSS on a real case. 
nal validation decision, different combination rules and forms of the discounting decreasing function will be analyzed.

Finally, and this is where this validation method seems very interesting, important work on learning the evolution of $B B A s$ at different levels will be developed. Indeed, to reduce the computation time related to the validation and to "cleverly" limit moments of validation, the issue of validation time choices and agents probed (i.e. type, number) remains important. At the Validation-Agent level, interest in the reliability of Parameter-Agents may also be important: the analysis of the possible discounting or reinforcement of some of them could appear significant.

As a conclusion, the global evidential calibration system provides excellent results. Future work will address the development of a evidential heuristic in order to provide a DSS totally based on belief functions. The very good results in forensic entomology could also allow the spread of the architecture in other areas, e.g. logistics or traffic flow management. This work is currently under development.

\section{Acknowledgments.}

This work is financed by the Norbert Ségard foundation. The authors thank Philippe Kubiak (LAGIS-École Centrale of Lille) and Damien Charabidze (Institute of forensic medicine of Lille) for their support.

\section{References}

[1] Ferber, J.: Multi-Agent Systems: An Introduction to Distributed Artificial Intelligence. Addison-Wesley Professional (February 1999)

[2] Banks, J., Carson, J., Nelson, B.L., Nicol, D.: Discrete-Event System Simulation. 5th edn. Prentice Hall (August 2009)

[3] Shafer, G.: A Mathematical Theory of Evidence. Princeton University Press, Princeton, New Jersey (1976)

[4] Smets, P., Kennes, R.: The transferable belief model. Artificial Intelligence 66(2) (1994) 191-234

[5] Malpica, J., Alonso, M., Sanz, M.: Dempster-shafer theory in geographic information systems: A survey. Expert Systems with Applications 32(1) (2007) $47-55$

[6] Mercier, D., Cron, G., Denœux, T., Masson, M.H.: Decision fusion for postal address recognition using belief functions. Expert Systems with Applications 36(3) (2009) 5643-5653

[7] Dempster, A.: Upper and lower probabilities induced by multivalued mapping. Annals of Mathematical Statistics AMS-38 (1967) 325-339 
[8] Kennes, R.: Computational aspects of the möbius transformation of graphs. IEEE Transactions on Systems, Man and Cybernetics 22 (1992) 201-223

[9] Yang, S.L., Fu, C.: Constructing confidence belief functions from one expert. Expert Systems with Applications 36(4) (2009) 8537-8548

[10] Veremme, A., Dupont, D., Lefevre, E., Mercier, D.: Belief assignment on compound hypotheses within the framework of Transferable Belief Model. In: 12th International Conference on Information Fusion, FUSION'2009. (2009) 498-505

[11] Smets, P.: The canonical decomposition of a weighted belief. In: International Joint Conference Artificial Intelligence. (1995) 1896-1901

[12] Denœux, T.: Conjunctive and disjunctive combination of belief functions induced by non distinct bodies of evidence. Artificial Intelligence 172(2-3) (2008) 234-264

[13] Smets, P.: Belief functions: The disjunctive rule of combination and the generalized bayesian theorem. International Journal of approximate Reasoning 9(1) (1993) 1-35

[14] Mercier, D., Quost, B., Denœux, T.: Refined modeling of sensor reliability in the belief function framework using contextuel discounting. Information Fusion 9(2) (2008) 246-258

[15] Smets, P.: Analyzing the combination of conflicting belief functions. Information Fusion 8(4) (2007) 387-412

[16] Yager, R.: On the Dempster-Shafer framework and new combination rules. Information Sciences 41 (1987) 93-138

[17] Dubois, D., Prade, H.: Representation and combination of uncertainty with beleif functions and possibility measures. Computational Intelligence 4 (1988) 244-264

[18] Lefevre, E., Colot, O., Vannoorenberghe, P.: Belief function combination and conflict management. Information Fusion 3(2) (2002) 246-258

[19] Klein, J., Lecomte, C., Miché, P.: Hierarchical and conditionale combination of belief functions induced by visual tracking. International Journal of Approximate Reasoning 51(4) (2010) 410-428

[20] Smets, P.: Decision making in a context where uncertainty is represented by the belief functions. Belief functions in Business Decisions (2002) 17-61

[21] Wooldridge, M.: An Introduction to Multiagent Systems - Second Edition. John Wiley \& Sons (2009) 
[22] Sempo, G., Depickère, S., Amé, J.M., Detrain, C., Halloy, J., Deneubourg, J.L.: Integration of an autonomous artificial agent in an insect society: Experimental validation. In: SAB. (2006) 703-712

[23] Railsback, S., Lytinen, S., Jackson, S.: Agent-based simulation platforms: Review and development recommendations. SIMULATION 82(9) (September 2006) 609-623

[24] Niazi, M., Hussain, A., Kolberg, M.: Verification and validation of agent based simulation using vomas approach. In: Proceedings of the Third Workshop on Multi-Agent Systems and Simulation (MASS09). (2009)

[25] Morvan, G., Veremme, A., Dupont, D., Jolly, D.: Démonstration : étude du coût de l'observation de simulations orientées agent. In: Journés Francophones sur les Systèmes Multi-Agents (JFSMA 2009), Lyon, France (Octobre 2009)

[26] Bethlehem, J.: Applied Survey Methods: A Statistical Perspective. WileyBlackwell (2009)

[27] Morvan, G., Dupont, D., Kubiak, P.: A simulation-based model of abduction. In: ESM'2008, Le Havre (2008) 183-187

[28] Gutknecht, O., Ferber, J., Michel, F.: Integrating tools and infrastructures for generic multi-agent systems. In: Proceedings of the Fifth International Conference on Autonomous Agents, ACM Press (2001) 441-448

[29] Stinner, R.E., Gutierrez, A.P., Butler Jr, G.D.: An algorithm for temperature-dependant growth rate simulation. The Canadian Entomologist 106 (1974) 519-524

[30] Morvan, G., Jolly, D., Dupont, D., Kubiak, P.: A decision support system for forensic entomology. In: Proceedings of the $6^{\text {th }}$ EUROSIM congress. (2007)

[31] Martin, A., Osswald, C., Dezert, J., Smarandache, F.: General combination rules for qualitative and quantitative beliefs. Journal of Advances in Information Fusion 3, 2, 67-89 (2008) 